\title{
Herbig-Haro flows in 3D: the HH 83 jet $^{\star}$
}

\author{
T. A. Movsessian ${ }^{1}$, T. Yu. Magakian ${ }^{1}$, A. V. Moiseev ${ }^{2}$, and M. D. Smith ${ }^{3}$ \\ 1 Byurakan Astrophysical Observatory, 378433 Aragatsotn reg., Armenia \\ e-mail: tigmov@bao.sci.am; tigmag@sci.am \\ 2 Special Astrophysical Observatory, N.Arkhyz, Karachaevo-Cherkesia 369167, Russia \\ e-mail: moisav@sao.ru \\ 3 Centre for Astrophysics \& Planetary Science, University of Kent, Canterbury CT2 7NH, UK \\ e-mail:m.d.smith@kent.ac.uk
}

Received 8 February 2009 / Accepted 28 September 2009

\begin{abstract}
Aims. The kinematics of the HH 83 optical outflow, located in the L 1641 molecular cloud, are investigated.

Methods. Observations were carried out with the Fabry-Perot scanning interferometer on the 6-m telescope of the Special Astrophysical Observatory. The $\mathrm{H} \alpha$ emission line was scanned with a spectral resolution of $R=8200$.

Results. The radial velocity along the jet increases with distance from the source, confirming previous results. It also shows lower amplitude variations which are not correlated with intensity. Both the spatial width of the jet as well as the FWHM of the H $\alpha$ emission line in the jet tend to decrease with distance from the source. The velocity field across the jet demonstrates a decrease from the center to the edges as well as some evidence for a transverse velocity gradient. The blue-shifted bow shock is separated spatially and spectrally into two distinct features, divided by about $2^{\prime \prime}$ and $250 \mathrm{~km} \mathrm{~s}^{-1}$, accordingly.

Conclusions. Evidence is provided that these split features correspond to forward and reverse shocks caused by a rapid pressure increase as the jet begins a new oblique impact on the surrounding medium. Radial velocity variations lengthwise and transverse to the jet axis are discussed. Linear extrapolation of the jet velocity up to the location of the terminal shock region yields the radial velocity of the reverse jet shock. The data are consistent with an abrupt outburst about one thousand years ago which ejected material with total speeds of up to $400 \mathrm{~km} \mathrm{~s}^{-1}$.
\end{abstract}

Key words. stars: formation - ISM: jets and outflows - ISM: clouds

\section{Introduction}

The Herbig-Haro object HH 83 was first noted as an unusual nebulous object with a cross-like knotty structure on a deep UK Schmidt red plate (Reipurth 1985). Further investigation revealed that $\mathrm{HH} 83$ consists of a small reflection nebula associated with a well-collimated jet of 32" length (Reipurth 1989), which at $450 \mathrm{pc}$ distant (the usually adopted distance to the L 1641 cloud) is equal to $0.07 \mathrm{pc}$. Infrared observations in the field revealed a source located to the south-east of $\mathrm{HH}$ 83, well aligned with the jet axis (Reipurth 1989; Moneti \& Reipurth 1995). This optically invisible source coincides with IRAS 05311-0631 with a luminosity of $10.5 L_{\odot}$ (Reipurth 1989). It was detected in the radio at the VLA by Rodriguez \& Reipurth (1998) and is also associated with a weakly collimated molecular flow observed in CO (Bally et al. 1994). An optical polarization map of the reflection nebula confirmed that it is illuminated by the infrared source (Rolph et al. 1990).

At a separation of $105^{\prime \prime}$ from the base of the jet, a bow shock structure was found on $\mathrm{H} \alpha$ images but was not visible on [SII] images (Reipurth 1989). In addition, HH 83 possibly forms a parsec-scale outflow system with HH 84 which traces a projected distance of $1.46 \mathrm{pc}$ (Reipurth et al. 1997).

* Based on observations collected with the $6 \mathrm{~m}$ telescope of the Special Astrophysical Observatory (SAO) of the Russian Academy of Sciences (RAS), operated with the financial support of the Science Department of Russia (registration number 01-43.).
A spectral investigation of the jet revealed a pronounced velocity increase along its axis. In addition, the density and the excitation in the jet decrease with distance (Reipurth 1989).

It is interesting that the $\mathrm{HH} 83$ jet showed no signs of $\mathrm{H}_{2}$ or [FeII] emission during HST/NICMOS near-infrared imaging although narrow band filters were not utilised. However, faint line emission was detected by Podio et al. (2006). The HST data revealed the complex reflection nebula in the vicinity of the bright source star which represents the edges of a conical cavity excavated by the flow. Its features are smoothly curved with very sharp edges (Reipurth et al. 2000).

We present in this paper scanning Fabry-Perot (FP) interferometric observations of the $\mathrm{HH} 83$ system in $\mathrm{H} \alpha$ emission. This is a powerful method for obtaining kinematic information of extended emission nebulae such as $\mathrm{HH}$ objects and jets. The main goal of this investigation is to obtain full three dimensional (two spatial and one spectral) characteristics of the complete system. The observational method is described in Sect. 2. In Sect. 3, we analyse the radial velocity and spatial distributions in the jet and bow shock. A full discussion of the implications is provided in Sect. 4.

\section{Observations and data reduction}

Observations were carried out at the prime focus of the $6 \mathrm{~m}$ telescope of the Special Astrophysical Observatory (SAO, Russia) 


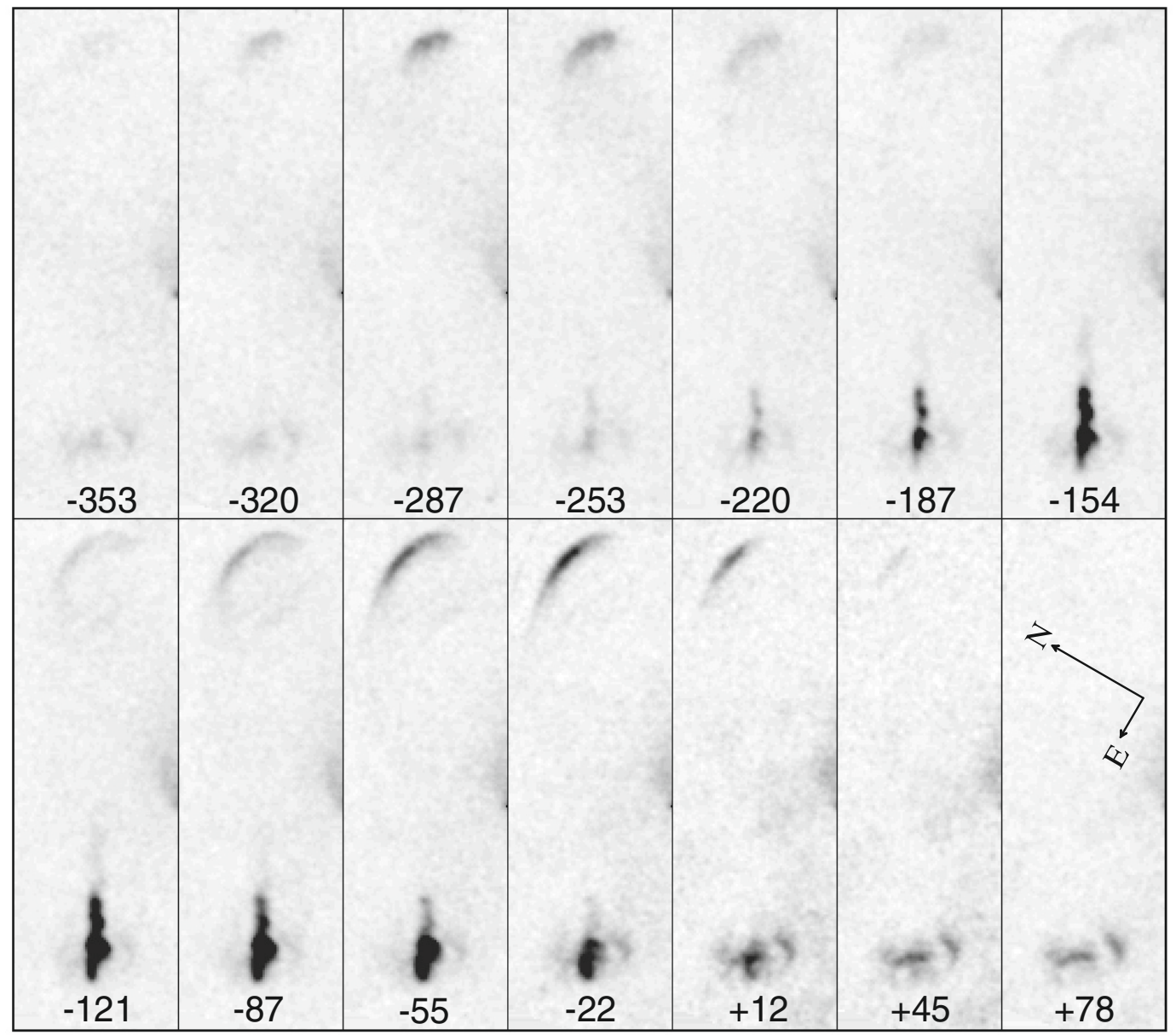

Fig. 1. Channel maps of the HH 83 region obtained by FP scanning of the $\mathrm{H} \alpha$ emission line. The heliocentric radial velocities in $\mathrm{km} \mathrm{s}^{-1}$ are presented at the bottom of each channel map. They correspond to the centers of the channels; each channel has a width of $32 \mathrm{~km} \mathrm{~s}^{-1}$. The size of each frame is $40 \times 112$ arcsec.

under good conditions (the seeing was $1^{\prime \prime}$ ). We used a scanning Fabry-Perot interferometer (IFP) placed in the parallel beam of the SCORPIO focal reducer (Afanasiev \& Moiseev 2005). The SCORPIO capabilities for IFP observations were also described by Moiseev (2002). The detector was a TK1024 $1024 \times$ 1024 pixel CCD array. Observations were performed with $2 \times$ 2 pixel binning to reduce the readout time, so $512 \times 512$ pixel images were obtained for each spectral channel. The field of view was $4.8^{\prime} \times 4.8^{\prime}$ with a scale of $0.56^{\prime \prime}$ per pixel. An interference filter with $F W H M=15 \AA$ centered on the $\mathrm{H} \alpha$ line was used for pre-monochromatization.

For our observations we used a Queensgate ET-50 interferometer operating in the 50th order of interference at the $\mathrm{H} \alpha$ wavelength, and providing a spectral resolution of $F W H M \approx$ $0.8 \AA$ (or $\sim 40 \mathrm{~km} \mathrm{~s}^{-1}$ ) for a range of $\Delta \lambda=13 \AA$ (or $\left.\sim 590 \mathrm{~km} \mathrm{~s}^{-1}\right)$, free from order overlapping. The number of spectral channels was 36 and the size of a single channel was $\Delta \lambda \approx$ $0.36 \AA\left(\sim 16 \mathrm{~km} \mathrm{~s}^{-1}\right)$.

We reduced our interferometric observations using software developed at the SAO (Moiseev \& Egorov 2008). After the primary data reduction, subtraction of night sky lines and wavelength calibration, the observational material represents "data cubes" where each point in the $512 \times 512$ pixel field contains a 36 channel spectrum. We removed the ghost images that appear in the plates of the interferometer using the algorithms described by Moiseev \& Egorov (2008). Optimal data filtering was performed by applying Gaussian smoothing over the spectral coordinate with $F W H M=1.5$ channels and spatial smoothing by a two-dimensional Gaussian with $F W H M=2$ pixels, by using the ADHOC software package ${ }^{1}$.

\section{Results}

The field covered by our FP images includes the entire HH 83 system: the jet, reflection nebula (actually the light reflected from the cavity walls) as well as the terminal shock region.

In Fig. 1, velocity channel maps are presented (to increase the $\mathrm{S} / \mathrm{N}$ ratio, each image was obtained by binning over two spectral channels). This illustrates that the object morphology depends strongly on the radial velocity (here and elsewhere in the paper the heliocentric velocities are used; to obtain the velocity with respect to the source one should subtract $21 \mathrm{~km} \mathrm{~s}^{-1}$ ).

The jet itself does not show prominent variations in its structure with the exception of the change of radial velocity along the axis, i.e. no new details appear or disappear according to the velocity.

The reflection nebula, as can also be seen from Fig. 1, is visible in all channels but becomes slightly brighter at low velocities. This is probably a result of the presence of the reflected $\mathrm{H} \alpha$ emission from the invisible stellar source.

The most interesting is the terminal shock region which is split into two discrete structures - a narrow and more elongated

1 The ADHOC software package was developed by J. Boulesteix (Marseille Observatory) and is publicly available on the Internet. 


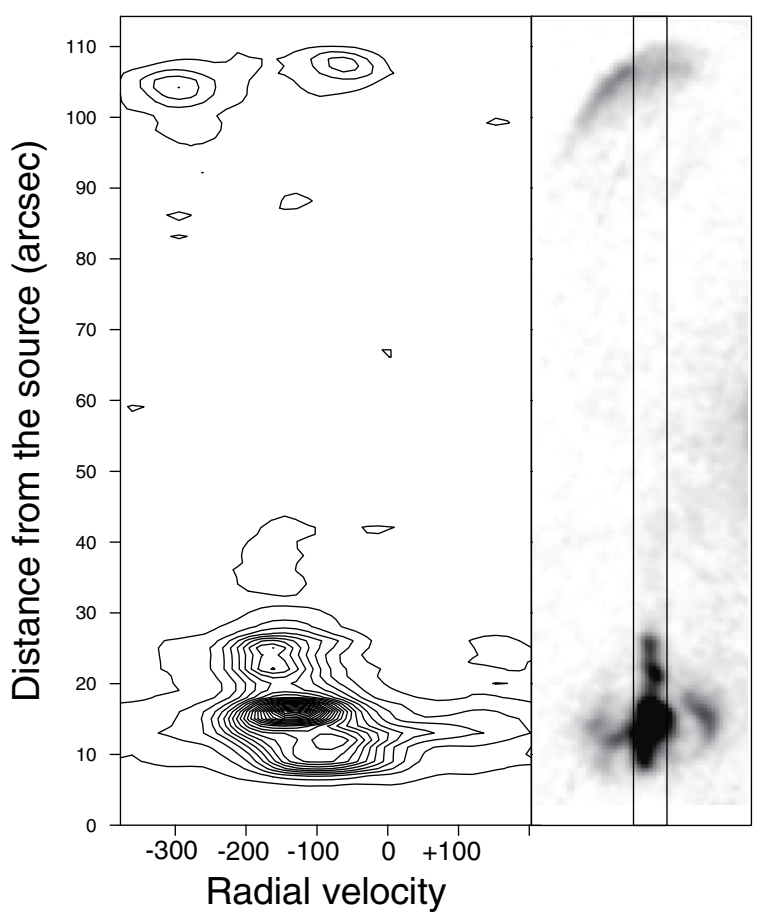

Fig. 2. Left panel: the position-velocity diagram for the HH 83 system. Right panel: the monochromatic image of the system in $\mathrm{H} \alpha$ showing the orientation of the pseudo-slit; its scale in arcsecs in both directions is the same as shown on the ordinate axis of the left panel.

bow outside of a clumpy and thick arc with a velocity separation between them of about $250 \mathrm{~km} \mathrm{~s}^{-1}$.

Below we discuss these parts of HH 83 separately.

\subsection{Velocity variations along the HH83 jet}

The position-velocity diagram along the flow axis with the slit width of 5 pixels (corresponding to $2.8^{\prime \prime}$ ) is presented in Fig. 2. It was created through the specific property of $3 \mathrm{D}$ data cubes which allows the construction of pseudo-slits with any selected direction and width. In the present case, the diagram was obtained by integrating the $\mathrm{H} \alpha$ intensity in each spectral channel across the slit width. The slit was made wide and oriented as shown in Fig. 2 to include all knots. To measure the distances of the knots from the source we used precise coordinates from Moneti \& Reipurth (1995) and Rodriguez \& Reipurth (1998); previous source positions given by Reipurth (1989) and Mundt et al. (1991) contain errors.

Our data provide detailed information about the velocity field in the entire HH 83 outflow system. In Fig. 3, the dependence of the heliocentric radial velocity along the HH 83 jet on the distance from the infrared source HH 83 IRS is presented. For better identification of knots, the intensity distribution along the jet is superimposed and knots D, F and G are labelled.

Both Figs. 2 and 3 display the obvious velocity increase along the HH 83 jet, first found and discussed by Reipurth (1989), and clearly show the split of the terminal shock region into two separate velocity structures. The velocity field in the jet part of the outflow is in agreement with the long-slit data of Reipurth (1989).

In Fig. 3, the straight line connects the velocity trend in the jet with the high velocity filament in the terminal shock region. One can note that such a linear extrapolation of the velocity up to the terminal shock region indeed is the good description of

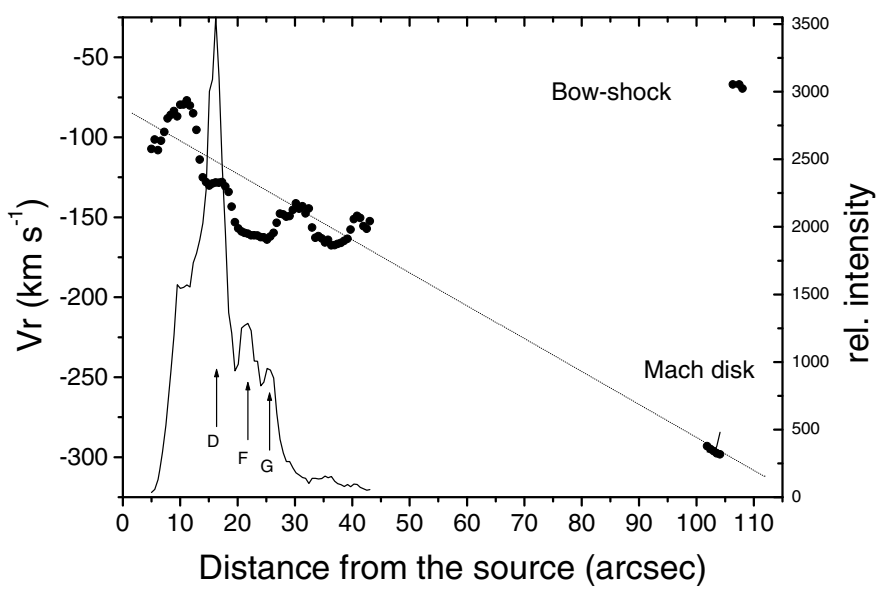

Fig. 3. The heliocentric radial velocity distribution (filled circles) along the HH 83 system versus the angular distance from the source. The working surface is separated into Mach disk and bow-shock structures. The positions of knots D, F, and G are identified by arrows on the superposed plot of the intensity of $\mathrm{H} \alpha$ emission (shown by thin line). The straight line extrapolates the jet velocity to the Mach disk, which is well separated from the bow shock in velocity.

the velocity field in this structure. We will discuss this region in more detail in Sect. 3.3.

Besides the general increase of the radial velocity with distance from the source, we note the smaller amplitude variations along the jet axis. As can be seen from Fig. 3, there is no significant correlation between the velocity and the emission intensity in the jet. Since the line emission depends on the shock properties and not on the velocity of the gas in the shock, this is not surprising.

On the other hand, these velocity variations in the jet are very important since they are related to the inner shocks in the jet. To estimate the shock velocity, we followed the approach of Hartigan et al. (2001). Correcting for the inclination angle of $45^{\circ}$ (Reipurth 1989), we obtain the true space velocities of the emitting material in the jet (assuming, of course, that the gas really is moving along the jet axis). Taking into account also the general trend of the velocity increase, we get the entire range of the space velocity variations in the jet as $\sim 115 \mathrm{~km} \mathrm{~s}^{-1}$. We note that this is a somewhat higher value than $100 \mathrm{~km} \mathrm{~s}^{-1}$, obtained for HH 111 by Hartigan et al. (2001). This conforms with the higher level of ionization of HH 83 (Podio et al. 2006). In addition, from Fig. 3 it is evident that the largest amplitude velocity variations are observed in the brightest part of the jet, i.e. near knots C-D-F-G, and become lower further out. This again can be compared with the prominent decrease of $n_{\mathrm{e}}, x_{\mathrm{e}}$ and $T_{\mathrm{e}}$ between knots $\mathrm{C}$ and $\mathrm{F}$, as shown by Podio et al. (2006).

\subsection{The $\mathrm{HH} 83$ jet in the transverse direction}

Our data show that the jet is broader than the seeing FWHM. This means that it is spatially resolved. The good spatial sampling allows us to accurately fit the spatial profiles along the restored image of the jet in $\mathrm{H} \alpha$, measuring in this way the $\mathrm{HH} 83$ jet width. The results, displayed in Fig. 4, can be compared with the measurements of Mundt et al. (1991) which were done for the [SII] line. Although one can come to the conclusion that in $\mathrm{H} \alpha$ the jet is slightly wider than in [SII], it is of little significance because our data were not deconvolved. However, the definite narrowing of the jet with distance from the source can be noted, which is not the case for the $[\mathrm{SII}]$ emission. 


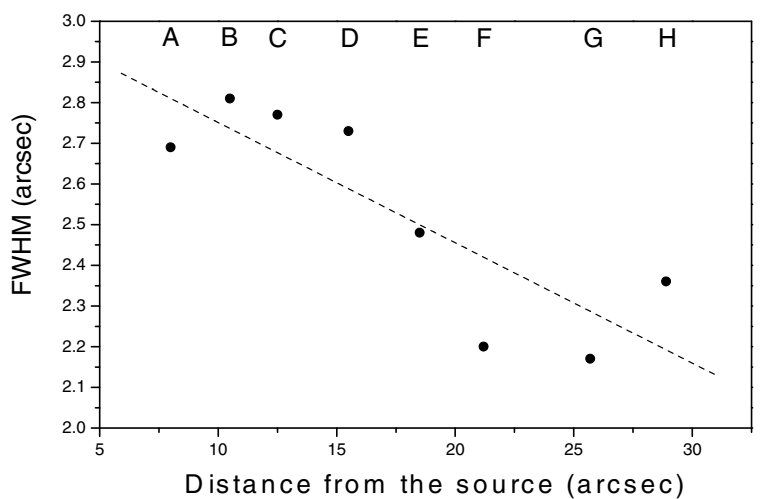

Fig. 4. The spatial FWHM of the HH 83 outflow (without deconvolution) plotted against the distance from the source. The general trend is shown by the dashed line.

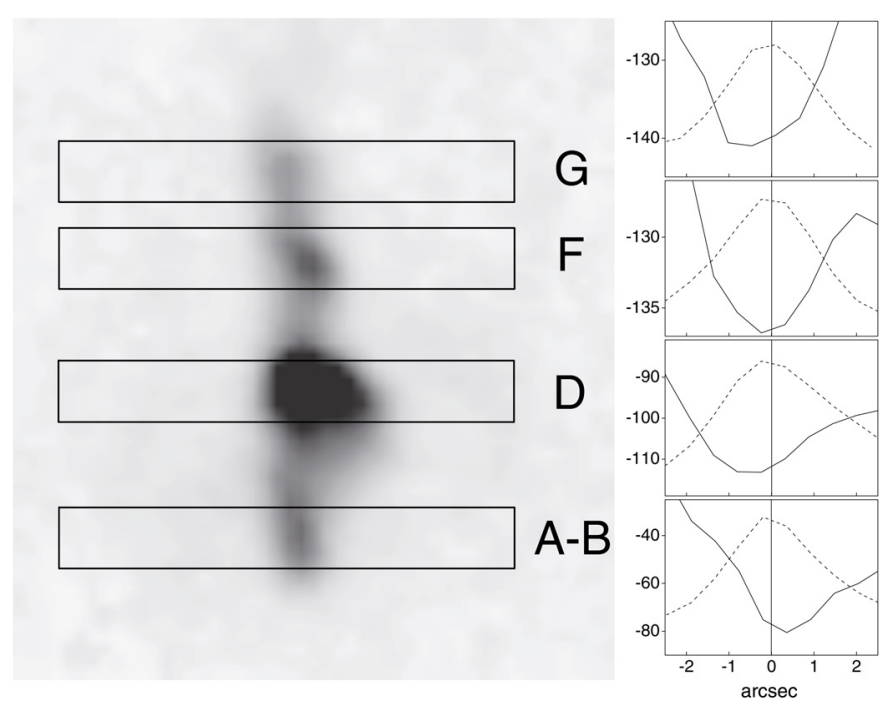

Fig. 5. The positions of pseudo-slits traced across knots A-B, D, F and $\mathrm{G}$ of the HH 83 jet (left panel). The size of each one of pseudo-slits is $2.8 \times 20.3^{\prime \prime}$. The corresponding heliocentric radial velocity distributions with superimposed intensity distributions (which are normalized to maximum and are shown by dashed lines) are shown on the right. The abscissae show the distance from the intensity peaks in arcseconds, and ordinates - velocities in $\mathrm{km} \mathrm{s}^{-1}$.

To measure the radial velocity changes across the $\mathrm{HH} 83$ jet, we traced several pseudo-slits, crossing the jet in knots $\mathrm{C}, \mathrm{D}$, $\mathrm{F}$ and G. Their width was set to five pixels (or $2.8^{\prime \prime}$ ) to increase the signal-to-noise ratio. Radial velocities were measured by Gaussian fitting of each profile along the pseudo-slit. As expected, the velocity fields obtained show a peak of the negative radial velocity near the jet axis and a decline moving to both sides (see, e.g., Movsessian et al. (2007) for another observational example and Beck et al. (2007) for a theoretical discussion). As can be seen from Fig. 5, where we show the positions of pseudo-slits and the velocity diagrams, in three cases the minimum of the negative velocity is shifted in the north-east direction from the jet axis (actually, since the axis of the not-straight jet is difficult to define, we used for each knot the local maximum of intensity as the zero-point): in knot D by $0.44^{\prime \prime}$, F by $0.34^{\prime \prime}$ and $\mathrm{G}$ by $0.52^{\prime \prime}$. In knot $\mathrm{C}$ the situation is reversed: the shift is $0.42^{\prime \prime}$ in the opposite direction.

Somewhat similar shifts have been found in the work of Coffey et al. (2007). However, we are not sure about the

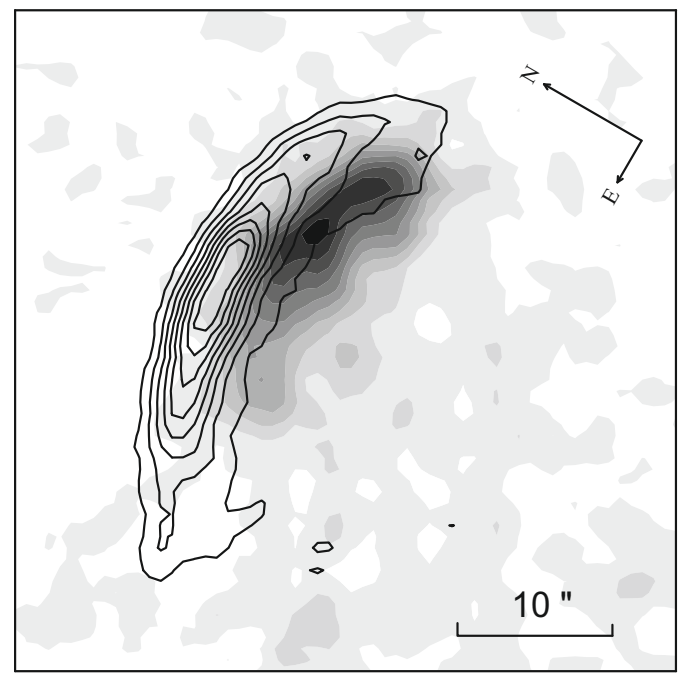

Fig. 6. The working surface of the HH 83 outflow, split into highvelocity (grey scale) and low-velocity (contoured) structures. These features are obtained by integrating the intensities in Gaussian-fitted components in the range of $\pm 25 \mathrm{~km} \mathrm{~s}^{-1}$ around the center velocity of $-295 \mathrm{~km} \mathrm{~s}^{-1}$ for the high-velocity component and $-68 \mathrm{~km} \mathrm{~s}^{-1}$ for the low-velocity one. The contours are drawn with a step of $10 \%$ of the peak intensities of the components.

rotational interpretation of the shift in the case of $\mathrm{HH} 83$. We will discuss this velocity trend in more detail in Sect. 4.

\subsection{The terminal shock region}

As mentioned above, our data show that the terminal shock region in the HH 83 system is divided into two distinct components which are well separated spectrally as well as spatially.

Spectral Gaussian fitting of the two components allows us to obtain the velocity field in each structure separately and to restore images in both components. It is clear from Fig. 6 that these two structures are shifted spatially by about $2^{\prime \prime}(\sim 1000 \mathrm{AU})$ and the narrow bow with low radial velocity is located in front of the knotty and more compact high velocity structure. One should also note that in the case of $\mathrm{HH} \mathrm{83,} \mathrm{the} \mathrm{bow} \mathrm{shock} \mathrm{region} \mathrm{is} \mathrm{un-}$ usually asymmetric about the jet axis: its northern wing is much brighter than the southern one. However, this asymmetry can be caused either by various degrees of absorption in the dark cloud or by an anisotropic distribution of the ambient medium.

Besides the evident division of both components in velocity, it should be noted that the high velocity component does not show significant variation of radial velocity in the transversal direction, while in the narrow low velocity structure the radial velocity decreases towards its wing from $-70 \mathrm{~km} \mathrm{~s}^{-1}$ near the apex to $-40 \mathrm{~km} \mathrm{~s}^{-1}$ near the edge of the bow-shaped structure.

\subsection{Line profiles}

The $\mathrm{H} \alpha$ emission in the $\mathrm{HH} 83$ jet mainly shows simple singlepeaked profiles. The exception is knot $\mathrm{C}$ where this emission is split into two components with radial velocities of -160 and $-70 \mathrm{~km} \mathrm{~s}^{-1}$. Some traces of the high-velocity component are also detected in knots $\mathrm{A}$ and $\mathrm{B}$. It is interesting that just near knot $\mathrm{C}$ a filament of the reflection nebula crosses the jet.

Inspection of the line profiles in other parts of the reflection nebula shows that their spectra are not purely continuum, and weak (compared to the jet) $\mathrm{H} \alpha$ emission also exists in this 


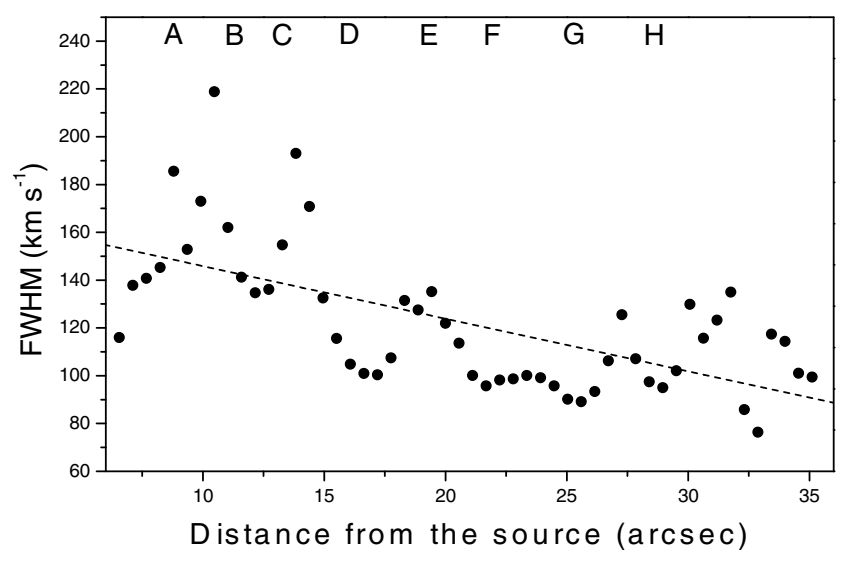

Fig. 7. The $\mathrm{H} \alpha$ line FWHM distribution along the $\mathrm{HH} 83$ jet (dots). The overall trend is approximated by a straight dashed line. The positions of knots are marked by letters.

region. However, contrary to the jet emission, the radial velocity of $\mathrm{H} \alpha$ in scattered light is about $+40 \mathrm{~km} \mathrm{~s}^{-1}$ and its $F W H M$ is $400 \mathrm{~km} \mathrm{~s}^{-1}$. This fully confirms the reflection origin of the $\mathrm{H} \alpha$ emission in the nebula.

The $F W H M$ variations of the $\mathrm{H} \alpha$ emission along the jet axis are presented in Fig. 7. As mentioned above, at the base of the jet the existence of the second, high-velocity component is evident. This component was fitted separately and excluded from the measurements of the general $F W H M$ of $\mathrm{H} \alpha$ in the jet. The significant variations of the $F W H M$ can be seen at the beginning of the jet although the main trend, distinct in Fig. 7, is a general decrease of the FWHM with distance from the source. This is consistent with a gradual decrease in the strength of shocks, as described in Sect. 3.1.

\section{Discussion and conclusion}

The increase of the jet velocity with distance from the source was discussed in detail by Reipurth (1989). His data led to the conclusion that this acceleration is not linear but decreases near the jet end. Our observations (see Fig. 3) are in agreement with Reipurth's data although our detection of a high-velocity component in the terminal shock region allows us to describe the general trend of radial velocity in the HH 83 flow as nearly linear, with superimposed variations of total amplitude of about $80 \mathrm{~km} \mathrm{~s}^{-1}$.

Several of the early scenarios raised and discussed by Reipurth can now be discounted, also taking into account the general progress in the understanding of stellar outflows. The practically linear law of the HH 83 outflow velocity field, which was extended by our data to a distance of $105^{\prime \prime}$, is consistent with the idea of a time dependent outflow. Moreover, it seems that such a linear dependence over about a thousand years points to an abrupt outburst with a subsequent decrease of ejection - a suggestion made by Reipurth (1989) as another possible scenario for $\mathrm{HH} 83$ and supported by the recent discussions that connect $\mathrm{HH}$ ejections to FU Ori type stellar activity (Reipurth \& Bally 2001). Such an approach combines the nearly instantaneous release of energy, typical of the FU Ori phenomenon, with a gradual fading of the outflow on timescales of the order of $10^{3}$ years. The total jet speed probably reaches $400 \mathrm{~km} \mathrm{~s}^{-1}$, assuming an angle of $45^{\circ}$ to the line of sight.

A scenario based on a steady jet flow over timescales exceeding the dynamical time cannot be excluded. However, this requires a gradual acceleration mechanism for which there is no obvious candidate. Alternatively, a high-speed undetected jet could be present. In this case, a neutral core jet shocks and accelerates surrounding material to produce the visible jet and itself becomes visible only when directly impacting at the bow shock.

Less pronounced but still complex variations of the velocity and $F W H M$ in the jet knots are very intriguing. They, perhaps, reflect the history of the smaller scale ejections from the central star, which created the separate knots in the jet. However, as was also mentioned above, the jet knots could be recently formed shock structures, as smooth velocity waves steepen within the jet. In this case, high knot pressure can produce a rapid transverse expansion. Note that, close to the source, radial velocities in the bright inner jet suggest total inner jet speeds of only $150 \mathrm{~km} \mathrm{~s}^{-1}$, which is not much in excess of the $F W H M$ for the same jet section. Hence, the jet may widen considerably and the present set of knots will fade rapidly rather than be seen to propagate down the jet, unless the motions giving rise to the velocity width are confined.

Velocity variations detected transverse to the jet axis can, in principle, be considered to be the result of two combined phenomena. First, the velocity drop near both edges of the jet may be due to a turbulent interaction with the ambient medium or with an enveloping wind. Secondly, the shift between the velocity and intensity peaks can be interpreted as due to an additional azimuthal velocity component. Such azimuthal components are often taken to be a signature of jet rotation. Evidence for rotation has been found for several jets (see, e.g., Woitas et al. 2005; Coffey et al. 2007, and references there). In our case, however, one should keep in mind that the shifts shown in Fig. 5 are detected at rather large distances from the source compared with observations of Coffey et al. (2007), and are not consistent with a rotation hypothesis, being directed in opposite directions. Thus, we do not suggest evidence for jet rotation, especially because alternate interesting explanations exist, e.g. see Soker (2005, 2007); besides, for the large distances from the exciting source it is more likely that any evidences of rotation will be lost in the turbulent structures inherent to the jet (Beck et al. 2007). Moreover, the transverse velocity gradient across the jet may be the result of a non-circular jet in which one side is aligned closer to the line of sight than the other. Of course, observational tests of such ideas require much higher spatial resolution than achieved so far.

The split of the emission line in knot $\mathrm{C}$ as well as the general decrease of $F W H M$ with distance from the source can be the result of higher turbulent motion near the source where the jet passes the cavity; then it becomes more laminar when propagating out of the cloud.

The bow shock structure is represented by two divided structures, separated spatially as well as kinematically. The extrapolation of the jet velocity gradient up to the terminal shock region, where the jet rams into the ambient medium, strongly supports the classical working surface concept where an advancing bow shock is followed by a Mach disk through which the jet flow is halted by a reverse shock.

However, we suggest here that the shock configuration is non-steady. The forward shock appears to be just entering a thick arc of cold stationary molecular gas identified in CO (Bally et al. 1994). This arc is roughly $110^{\prime \prime}$ from HH 83 -IRS and runs orthogonal to the jet. Here, the reverse shock is oblique and, hence, the common term Mach disk such as used by Morse et al. (1992) is not an apt description. Furthermore, the separation of the forward and reverse shocks is not predicted from hydrodynamic numerical simulations (Blondin et al. 1989). To 
explain the separation, we propose that the shocks have recently strengthened due to the obstructing arc, resulting in a pressure increase within the shocked layer. The adjustment of the two shocks to this increase in pressure then leads to an expanding layer, and hence a detectable spatial separation. The lack of detectable [SII] emission may then also be attributed to the truncated nature of the post-shock cooling layers in which incomplete cooling occurs. For this to occur over an angular distance of two arcseconds at a distance of $420 \mathrm{pc}$ requires a cooling length in excess of $1.2 \times 10^{16} \mathrm{~cm}$. The atomic cooling time scale at $10000 \mathrm{~K}$ is of the order of $10^{11} / n$ s where $n$ is the atomic density in units of $\mathrm{cm}^{-3}$, e.g. Smith \& Rosen (2003). Therefore, taking a speed of $300 \mathrm{~km} \mathrm{~s}^{-1}$ (the tangential component of the gas speed approaching the shock surface), a truncated shock is expected for densities below $250 \mathrm{~cm}^{-3}$. In comparison, the bow shock and putative Mach disk in HH34S are separated by $\sim 5 \times 10^{16} \mathrm{~cm}$, where magnetic field pressure or cool neutral atomic gas were suggested to occupy the gap (Morse et al. 1992).

The highest jump in (projected) velocity of $250 \mathrm{~km} \mathrm{~s}^{-1}$ occurs directly at the projected interface of the two shock waves. This also suggests that the interaction is via two deflecting oblique shocks and that the post-shock ambient gas is deflected in the opposite direction transverse to the jet gas.

A problem remains of how the thick arc has come to obstruct the jet. The bipolar outflow and star are probably quite old with signs of having blown out through the cloud. Hence, for the jet to now still be obstructed would suggest that the jet orientation has changed, gradually eroding the cloud in new directions.

We detected the $\mathrm{H} \alpha$ emission in reflected light from the cavity walls, which indicates that the illuminating source is an emission line star deeply embedded in the dark cloud. It is very probably a classical T Tauri star and not a Herbig AeBe star, with its high IRAS luminosity at this early evolutional state largely derived from accretion.

Acknowledgements. The authors are grateful to Bo Reipurth for the discussion of the manuscript and many helpful comments. They also thank the referee whose valuable suggestions led to a significantly improved paper. This project was mainly supported by INTAS grant 00-00287 and partially supported by ANSEF grant No. PS103-01.

\section{References}

Afanasiev, V. L., \& Moiseev, A. V. 2005, Astron. Lett., 31, 193 Bally, J., Castets, A., \& Duvert, G. 1994, ApJ, 423, 310

Beck, T. L., Riera, A., Raga, A. C., \& Reipurth, B. 2007, AJ, 133, 1221

Blondin, J. M., Konigl, A., \& Fryxell, B. A. 1989, ApJ, 337, L37

Brown, A., Drake, S. A., \& Mundt, R. 1985, in Radio Stars, R. M. Hjellming, D. M. Gibson ed. (Dordrecht: Reidel), 105

Coffey, D., Bacciotti, F., Ray, T. P., Eislöffel, J., \& Woitas, J. 2007, ApJ, 663, 350

Cohen, M., \& Kuhi, L. V. 1979, ApJS, 41, 743

Hartigan, P., Morse, J. A., Reipurth, B., Heathcote, S., \& Bally, J. 2001, ApJ, 559, L157

Magakian, T. Yu., Movsessian, T. A., Afanasiev, V. L., \& Burenkov, A. N. 1989, Sov. Astr. Lett., 15, 53

Moiseev, A. V. 2002, Bull. SAO, 54 [arXiv: astro-ph/0211104]

Moiseev A. V., \& Egorov, O. V. 2008, Astrophys. Bull., 63, 181

Movsessian, T., Boulesteix, J., Gach, J.-L., \& Zaratsian, S. 2000, Baltic Astron., 9,652

Movsessian, T. A., Magakian, T. Y., Bally, J., et al. 2007, A\&A, 470, 605

Moneti, A., \& Reipurth, B. 1995, A\&A, 301, 721

Morse, J. A., Hartigan, P., Cecil, G., Raymond, J. C., \& Heathcote, S. 1992, ApJ, 399, 231

Mundt, R., \& Fried, J. W. 1983, ApJ, 274, L83

Mundt, R., Brugel, E. W., \& Bührke, T. 1987, ApJ, 319, 275

Mundt, R., Ray, T., \& Bührke, T. 1988, ApJ, 333, L69

Mundt, R., Ray, T. P., Bührke, T., Raga, A. C., \& Solf, J. 1990, A\&A, 232, 37

Mundt, R., Ray, T. P., \& Raga, A. C. 1991, A\&A, 252, 740

Podio, L., Bacciotti, F., Nisini, B., et al. 2006, A\&A, 456, 189

Reipurth, B. 1985, A\&AS, 61, 319

Reipurth, B. 1989, A\&A, 220, 249

Reipurth, B., \& Bally, J. 2001, ARA\&A, 39, 403

Reipurth, B., Bally, J., \& Devine, D. 1997, AJ, 114, 2708

Reipurth, B., Yu, K. C., Heathcote, S., Bally, J., \& Rodríguez, L. F. 2000, AJ, 120,1449

Rodríguez, L. F., \& Reipurth, B. 1998, RMxAA, 34, 13

Rodríguez, L. F., Cantó, J., Torrelles, J. M., Gómez, J. F., \& Ho, P. T. P. 1992, ApJ, 393, L29

Rodríguez, L. F., Cantó, J., Torrelles, J. M., et al. 1994, ApJ, 427, L103

Rolph, C. D., Scarrott, S. M., \& Wolstencroft, R. D. 1990, MNRAS, 242, 109

Smith, M. D., \& Rosen, A. 2003, MNRAS, 339, 133

Soker, N. 2005, A\&A, 435, 125

Soker, N. 2007 [arXiv: astro-ph/0703474v1]

Wilner, D. J., Ho, P. T. P., \& Rodríguez, L. F. 1996, ApJ, 470, L117

Woitas, J., Bacciotti, F., Ray, T. P., et al. 2005, A\&A, 432, 149 